\title{
A comparative analysis of direct LDL-C assay and Friedewald's formula in subjects of ischemic heart disease \& stroke in a tertiary care centre
}

\author{
Bijoya P. Chatterjee ${ }^{1}$, Sandip Sendhave,", Amit Kakaiya ${ }^{3}$, Prasanta Chatterjee Biswas ${ }^{4}$ \\ ${ }^{1}$ Tutor, ${ }^{2,3}$ Assistant Professor, Dept. of Biochemistry, M P Shah Medical College, Jamnagar, Gujarat, ${ }^{4}$ Associate Professor, Dept. \\ of Quantitative Research and Analytics, Marwadi University, Rajkot, Gujarat, India
}

*Corresponding Author: Sandip Sendhav

Email: sandip.sendhav@yahoo.in

Received: $30^{\text {th }}$ May, 2018

Accepted: $5^{\text {th }}$ July, 2018

\begin{abstract}
Introduction: Among the various risk factors for the development of Ischemic heart diseases (IHD) as well as stroke is elevated low density lipoprotein cholesterol (LDL-C) levels. This makes accurate reporting of LDL-C crucial in the management of IHD. On one hand estimation of LDL-C by direct method is available, but it is expensive and on the other hand the Friedewald's formula is most commonly employed but a method with limitations.

Aim: Therefore the aim of this study is to evaluate the correlation between calculated LDL-C with direct LDL-C method and analyse their appropriateness.

Materials and Methods: This study was a hospital based cross-sectional study. LDL-C was measured by both Friedewald's formula (FF) and direct LDL method in 501 participants between the age group of 30-70 years. Paired $t$-test was used to test the difference in LDL concentration obtained by a direct method and Friedewald's formula. The level of significance was taken as $\mathrm{P}$ $<0.05$.

Results: The mean and standard deviation (SD) of LDL-C estimated by both direct assay and FF showed a significant difference $(p<0.05)$ at TG ranges of $<150$ and $151-399 \mathrm{mg} / \mathrm{dL}$ respectively but there is no significant difference $(\mathrm{p}>0.05)$ at TG range $>400 \mathrm{mg} / \mathrm{dL}$. There was a discrepancy of 37 out of 501participants $(7.38 \%)$ classified as cardiac risk groups by the two methods used. A significant correlation between LDL-C levels obtained by FF and direct methods, $(p$ value $<0.05, \mathrm{r}$ value $=0.8101, \mathrm{CI}=$ 0.7777 to 0.8382 ) was observed.

Conclusion: The study concludes that both methods can be employed depending on TG levels. Direct LDL estimation appears to be unreliable at TG concentrations $>400 \mathrm{mg} / \mathrm{dL}$ but useful when sample is collected from a non-fasting participants.
\end{abstract}

Keywords: Low density lipoprotein, Ischemic heart disease, Stroke.

\section{Introduction}

Elevated lipids have been suggested as a risk factor for developing Ischemic heart disease (IHD) and stroke. The association between total cholesterol (TC) and risk of developing IHD has been long established by studies. ${ }^{1-3}$ Most of the cholesterol in circulation is carried by low density lipoprotein (LDL), and because LDL-cholesterol (LDL-C) is a modifiable risk factor for coronary heart disease, its routine measurement is recommended in the evaluation and management of hypercholesterolemia. The diagnosis and management of adults with hypercholesterolemia are principally targeting LDL-C levels in circulation. ${ }^{4}$ The serum LDL$\mathrm{C}$ concentrations used to classify adults for high risk of heart disease are: Desirable $<130 \mathrm{mg} / \mathrm{dL}$, Borderline high-risk 130-159 mg/dL, High risk >160 mg/dL. ${ }^{2}$ The goal for subjects with two or more risk factors (diabetes, family history, hypertension, cigarette smoking, low high density lipoprotein cholesterol (HDL-C)) is to achieve LDL-C of $100 \mathrm{mg} / \mathrm{dL}^{5}$ Therefore accurate and precise measurements of subjects LDL-C concentrations are necessary to appropriately identify individuals with hypercholesterolemia and to monitor the response of various treatments as well as lifestyle changes. ${ }^{6}$
The LDLs are spherical particles, having a hydrophobic oily core of cholesteryl ester and triglycerides (TG). These particles have a hydrophilic envelop of phospholipids, free cholesterol and apolipoproteins. On an average, LDL carries two thirds of the total cholesterol (TC) in serum. Each LDL particle contains one molecule of apolipoprotein B-100 (apo B-100), which is the main apolipoprotein of LDL, and the others are minor (apo E and apo C II). ${ }^{7}$ The reference method for determining LDL-C is $\beta$ quantification. ${ }^{2}$ It requires ultracentrifugation which requires large sample quantity. Besides it is a time consuming and expensive technique. Therefore, this method is not suitable for routine laboratory testing. ${ }^{8}$ Hence its use in routine clinical laboratories has limitations. In 1972, Friedewald et al. published a landmark report describing a formula to estimate LDL$\mathrm{C}$ as an alternative to ultra-centrifugation. ${ }^{9}$ As VLDL carries most of the circulating TGs, VLDL-C can be estimated from measured TGs divided by 5 for $\mathrm{mg} / \mathrm{dL}$ (TG/5). LDL-C is then calculated as TC minus HDL-C minus estimated VLDL-C [LDL-C=TC- (HDLC+VLDL-C)]. So the most commonly employed method in the determination of LDL-C in a clinical laboratory is done using the Friedewald's formula (FF). 
It needs no reagents so cost-effective. Although convenient, the FF has its limitations too which are:-

1. LDL-C $=$ TC- $($ HDL-C+VLDL-C $)$.This formula uses three independent quantifications which causes an imprecision in estimating LDL-C concentrations.

2. It is unreliable at $\mathrm{TG}$ concentrations $>400 \mathrm{mg} / \mathrm{dL}$ and,

3. An overnight fasting blood sample is required.

For these reasons NCEP recommended the development of accurate direct LDL-C methods. Earlier direct methods had limitations for general use. Recently, a new generation of homogeneous method capable of full automation has been introduced that uses specific reagents to selectively expose and directly measure the cholesterol associated with direct LDL-C. The aim of this study is therefore to compare homogenous enzymatic direct assay using autoanalyser with LDL-C obtained by FF in subjects with CVA, IHD and hypertension.

\section{Materials and Methods}

Participants of the study fell between 30-70 years of age of either sex with clinical findings, brain CTscan or MRI indicative of cerebral infraction or intracerebral hemorrhage were enrolled in this study. Patients in the same age range diagnosed of IHD and hypertension (HTN) were also enrolled in the study. ACS refers to a group of clinical symptoms that encompass clinical conditions ranging from unstable angina pectoris (USAP) to myocardial infarction (nonST-segment elevation myocardial infarction (NSTEMI) to ST-segment elevation myocardial infarction (STEMI). The presence of ACS was classified, based on a clinical diagnosis of AMI or USAP was made on the basis of classical symptoms, diagnostic electrocardiogram (ECG) changes, cardiac injury enzymes and/or echocardiographic (ECG) evidences. The patients with any underlying diseases especially liver disease, renal disease, and hypothyroidism, taking anti lipid and sympathomimetic drugs, and the patients in whom the cerebral hemorrhage was secondary to cerebral tumor, trauma or previous coagulation disorders, pregnant women were excluded from the study. Data was obtained from the lipid profile analysis performed in the Clinical Biochemistry Laboratory of M. P. Shah Government Medical College and Guru Gobindsingh Government Hospital, Jamnagar. This cross sectional study was carried after receiving permission from the Institutional Ethical Committee. Informed consents were obtained from all participants.

Biochemical Tests: Five $\mathrm{ml}$ of blood sample was collected after 10-12 hours of overnight fasting in plain vials. The blood samples were allowed to clot. The serum was separated by centrifugation for 20 minutes at $5000 \mathrm{rpm}$ and the following parameters estimated:-

1. TC by Enzymatic endpoint CHOD-PAP method.
2. TG by Enzymatic Glycerol Phosphate Oxidase/ Peroxidase method.

3. HDL-C by Homogenous Enzymatic Direct Assay.

4. LDL-C by Homogenous Enzymatic Direct Assay.

5. Calculated VLDL-Cholesterol (VLDL-C) obtained by Friedewald's formula.

6. Calculated LDL-C obtained by Friedewald's formula.

TC (CHOD-PAP Method): Serum TC was determined after enzymatic hydrolysis and oxidation. The indicator was a quinoneimine dye formed from hydrogen peroxideand 4-aminoantipyrine in the presence of phenol and peroxidase. ${ }^{10}$

TG (GPO-PAP Method): TG was determined after enzymatic hydrolysis with lipases. Hydrogen peroxide, 4-aminophenazoneand 4-cholorophenol reacted to form quinoneimine dye by the catalytic action of enzyme peroxidase. ${ }^{11}$

HDL-C (Homogeneous Enzymatic Direct Method): The antigen- antibody complex is formed with all serum lipoproteins (LDL-C, VLDL-C, and chylomicrons) except HDL-C. This prevented them from reacting with enzymes cholesterol esterase and cholesterol oxidase. HDL-C was then determined by enzymes cholesterol esterase and cholesterol oxidase. ${ }^{12}$ LDL-C (Homogeneous Enzymatic Direct Method): The LDL-C Direct estimation was done by homogeneous assay. LDL-C was converted to hydrogen peroxide in the first step. Hydrogen peroxide so formed reacted with 4-aminoantipyrin to give a blue coloured complex. The absorbance of this blue coloured complex gave the LDL-C concentration in the sample upon calculation. ${ }^{13}$

VLDL-C and calculated LDL-C by Friedewald's Formula (FF): The FFis given by: LDL-C $=\mathrm{TC}-$ HDL-C -VLDL-C (mg/dL). In this formula TG/5 gives a measure of VLDL-C because VLDL carries most of the circulating TGs, and so VLDL-C was estimated from measured serum $\mathrm{TG}(\mathrm{TG} / 5 \mathrm{in} \mathrm{mg} / \mathrm{dL}){ }^{9}$

\section{Statistical Analyses}

Data thus collected was categorized into 3 groups based on the serum TG levels. Group I (TG $\leq 150$ $\mathrm{mg} / \mathrm{dL}$ ), Group II (TG 151-399 mg/dL) and Group III ( $\mathrm{TG} \geq 400 \mathrm{mg} / \mathrm{dL}$ ). Statistical analysis was done using student $t$ test and Pearson's correlation coefficient. $\mathrm{p}$ value $<0.05$ were considered to be statistically significant. All statistical analysis was carried out using the software known as a Graphpad prism version 5.

\section{Results}

In present study, 501 subject participated out of which 29\% subjects are from 25-45 years age group, $59 \%$ are from $46-65$ years age group and $12 \%$ subjects are from more than 65 year age group, moreover $60 \%$ are male and $40 \%$ are female (Table 1). 66\% subjects had a BMI greater than $25 \mathrm{Kg} / \mathrm{mt}^{2}$ (Table 1). The 
percentage error between calculated LDL-C and Direct LDL-C ranged from $2.56 \%$ to $-20.83 \%$ at different TG levels. The error was more at TG levels >400 mg\% (Table 2). The mean and standard deviation (SD) of LDL-C estimated by both direct assay and FF showed a significant difference $(\mathrm{p}<0.05)$ at $\mathrm{TG}$ ranges of $<150$ and 151- $399 \mathrm{mg} / \mathrm{dL}$ respectively but there is no significant difference $(\mathrm{p}>0.05)$ at $\mathrm{TG}$ range $>400$ $\mathrm{mg} / \mathrm{dL}$ (Table 3). Table 4 shows that there was a discrepancy of 37 patients classified as cardiac risk groups by the two methods used. In the current study,
37 out of a total of 501 patients constitute 7.38\%.Graph 1 showed significant correlation between LDL-C levels obtained by FF and direct methods, ( $p$ value <0.05, r value $=0.8101, \mathrm{CI}=0.7777$ to 0.8382$)$. Correlation between calculated and Direct LDL-C in different TG groups are given in table 5. There was statistically significant difference in the means of LDL-C levels obtained by the two methods at TC levels 100-199 $\mathrm{mg} / \mathrm{dL}(\mathrm{p}<0.05)$. This difference was not observed at TC levels $>200 \mathrm{mg} / \mathrm{dL}(\mathrm{p}>0.05)$ (Table 6).

Table 1: Demographic Characteristics of the study population

\begin{tabular}{|l|c|c|c|}
\hline Demographic Characteristics & Parameters & Total & Percentage (\%) \\
\hline \multirow{3}{*}{ Age(yrs) } & $25-45$ & 144 & 29 \\
\cline { 2 - 4 } & $46-65$ & 297 & 59 \\
\cline { 2 - 4 } & $>65$ & 60 & 12 \\
\hline \multirow{3}{*}{ Gender } & Male & 302 & 60 \\
\cline { 2 - 4 } & Female & 199 & 40 \\
\hline \multirow{2}{*}{ Dietary habit } & Veg & 470 & 94 \\
\cline { 2 - 4 } & Non-veg & 31 & 6 \\
\hline \multirow{3}{*}{ BMI (kg/mt $\left.{ }^{2}\right)$} & $18.5-22.9$ & 74 & 15 \\
\cline { 2 - 4 } & $23-24.9$ & 94 & 19 \\
\cline { 2 - 4 } & $\geq 25$ & 333 & 66 \\
\hline \multirow{2}{*}{ Obesity } & Non-Obese & 168 & 34 \\
\cline { 2 - 4 } & Obese & 333 & 66 \\
\hline Diagnosis & IHD & 169 & 34 \\
\cline { 2 - 4 } & CVA & 168 & 34 \\
\cline { 2 - 4 } & HTN & 164 & 33 \\
\hline
\end{tabular}

Table 2. Percentage error of the means of LDL-C estimated by two methods

\begin{tabular}{|l|c|c|c|c|}
\hline $\begin{array}{c}\text { TG range } \\
(\mathbf{m g} / \mathbf{d L})\end{array}$ & $\begin{array}{c}\text { Study subjects } \\
(\mathbf{n = 5 0 1})\end{array}$ & $\begin{array}{c}\text { Mean of Calculated LDL- } \\
\mathbf{C}(\mathbf{m g} / \mathbf{d L})\end{array}$ & $\begin{array}{c}\text { Mean of Direct LDL-C } \\
(\mathbf{m g} / \mathbf{d L})\end{array}$ & \% Error \\
\hline$\leq 150$ & 297 & 133.77 & 131.21 & 2.56 \\
\hline $151-399$ & 192 & 141.28 & 150.63 & -9.35 \\
\hline$\geq 400$ & 12 & 130.25 & 151.08 & -20.83 \\
\hline
\end{tabular}

The percentage error between Calculated LDL-C and Direct LDL-C ranged from $2.56 \%$ to $-20.83 \%$ at different TG levels. The error was more at TG levels $>400 \mathrm{mg} / \mathrm{dL}$.

Table 3: Comparison of LDL-C Direct with that of LDL-C calculated in each TG range

\begin{tabular}{|l|c|c|c|c|}
\hline Groups & $\begin{array}{c}\text { TG Range } \\
(\mathbf{m g} / \mathbf{d L})\end{array}$ & $\begin{array}{c}\text { Direct LDL-C } \\
(\mathbf{m g} / \mathbf{d L}) \mathbf{( M e a n} \pm \text { SD) }\end{array}$ & $\begin{array}{c}\text { Calculated LDL-C } \\
(\mathbf{m g} / \mathbf{d L})(\text { Mean } \pm \text { SD })\end{array}$ & P value \\
\hline I & $\leq 150 ; \mathrm{n}=297$ & $131.21 \pm 44.28$ & $143.77 \pm 48.11$ & $<0.05$ \\
\hline II & $151-399 ; \mathrm{n}=192$ & $150.63 \pm 46.04$ & $161.28 \pm 45.74$ & $<0.05$ \\
\hline III & $\geq 400 ; \mathrm{n}=12$ & $151.08 \pm 37.73$ & $146.25 \pm 51.38$ & $>0.26$ \\
\hline
\end{tabular}

Table 4: Classification of patients by the two methods of LDL-C measured

\begin{tabular}{|c|c|c|}
\hline LDL-C levels & By Direct assay (\%) & By Friedewald estimation (\%) \\
\hline$<130 \mathrm{mg} / \mathrm{dL}$ & $264(52.69)$ & $227(45.31)$ \\
\hline$>130 \mathrm{mg} / \mathrm{dL}$ & $237(47.31)$ & $274(54.69)$ \\
\hline Total no. of participants (n) & $501(100)$ & $501(100)$ \\
\hline
\end{tabular}

Table 5: Correlation between calculated and Direct LDL in different TG groups

\begin{tabular}{|l|c|c|c|}
\hline TG Ranges (mg/dL) & r Value & P Value & $\mathbf{9 5 \%}$ CI \\
\hline$\leq 150$ & 0.8542 & $<0.05$ & 0.8201 to 0.8823 \\
\hline $151-399$ & 0.7575 & $<0.05$ & 0.6898 to 0.8120 \\
\hline$\geq 400$ & 0.8339 & $<0.05$ & 0.4986 to 0.9522 \\
\hline
\end{tabular}


Table 6: Comparison of LDL-C Direct with LDL-C calculated in each total cholesterol (TC) range groups

\begin{tabular}{|l|c|c|c|c|}
\hline \multicolumn{1}{|c|}{ Groups } & $\begin{array}{c}\text { TC } \\
(\mathbf{m g} / \mathbf{d L})\end{array}$ & $\begin{array}{c}\text { Direct LDL-C } \\
(\mathbf{m g} / \mathbf{d L})\end{array}$ & $\begin{array}{c}\text { Calculated LDL-C } \\
(\mathbf{m g} / \mathbf{d L})\end{array}$ & P value \\
\hline I & $100-149$ & $87.13 \pm 17.30$ & $76.85 \pm 15.16$ & $<0.05$ \\
\hline II & $150-199$ & $117.40 \pm 27.84$ & $110.20 \pm 16.27$ & $<0.05$ \\
\hline III & $200-249$ & $144.54 \pm 26.10$ & $147.22 \pm 22.45$ & $>0.33$ \\
\hline IV & $\geq 250$ & $197.44 \pm 34.41$ & $201.49 \pm 24.67$ & $>0.30$ \\
\hline
\end{tabular}

Values of Direct LDL-C and Calculated LDL-C have been represented as Mean \pm SD

\section{Discussion}

According to the report of NCEP Adult Treatment Panel, LDL-C is the principle therapeutic target in the diagnosis and management of hypercholesterolemia. ${ }^{3}$ Hence, accurate reporting of LDL-C of utmost importance. In the present study, the percentage error between calculated LDL-C and Direct LDL-C is less when TG level $<400 \mathrm{mg} / \mathrm{dL}$. More number of patients can be screened out at high cardiac risk when calculated LDL-C estimation method is used as it not only reduces turnaround time but is also found to be highly cost effectiveness in comparison to direct estimation with the help of enzyme based reagents.

In the current study, on comparing Friedewald's estimation with direct assay for LDL-C a correlation co-efficient of 0.8101 (Graph 1) was observed. A significant difference was found in direct method and Friedewald's estimation for LDL-C, particularly in categories of different TG levels. It has been reported earlier that direct assays tend to have a negative bias at the low TG concentrations. ${ }^{14}$ This perhaps is instrumental in misclassification of hypercholesterolemia by showing subjects at a lower risk level. ${ }^{15}$ Despite the limitations, direct assays are accurate which makes them useful in following subjects with higher risk to IHD or stroke. ${ }^{15}$ Therefore it is used when the Friedewald's formula is not suitable (e.g. type 3 dysbetalipoprotieinemia and in fasting subject with hypertriglyceridemia). ${ }^{16,17}$ The NCEP Expert Panel observed that total analytical variability in calculated LDL-C fell between $2.7 \%$ and $6.8 \%$ for LDL-C concentrations between $100-225 \mathrm{mg} / \mathrm{dL}$ in well established laboratories. ${ }^{18}$ In routine laboratories, however the variability was much higher (around12\%). In order to reduce bias the Panel recommended development of better methods to reduce the error of FF as well as direct LDL-C estimation. ${ }^{18}$ Currently homogenous methods that are available for LDL-C estimations are programmable in automated analysers. Studies have shown that three out of five homogenous methods give results comparable to the Friedewald calculation and appear to meet NCEP performance criteria. ${ }^{19-23}$

According to NCEP, the desirable LDL-C limit is < $130-\mathrm{mg} / \mathrm{dL}$. In the current study, 37 out of a total of 501 subjects were found to be at high risk by Friedewald estimation and they had no cardiac risk by direct homogenous LDL-C measurement. If those 37patients as seen by Friedewald estimation were treated as high risk and were given cholesterol lowering agents that would be of no harm but have long term benefits. However, if those patients as seen by direct assay were classified as low risk and not actively managed, they could present with serious irreversible complication of IHD and or stroke. This could contribute in morbidity and mortality both. In a review study it was concluded that LDL-C direct assays could replace Friedewald calculation in those cases where calculation is unreliable, e.g; TG> $400 \mathrm{mg} / \mathrm{dL} .{ }^{24}$ Miller reported that the direct LDL-C results do not improve the performance of LDL-C calculated by Friedewald equation at TG concentrations of $<400 \mathrm{mg} / \mathrm{dL}{ }^{25-27}$ This is consistent with the current study where significant difference in LDL-C levels at lower TG levels (Table. 3 ) was seen. Studies have also recommended that nonHDL Cholesterol levels are more accurate than either the directly measured or the calculated LDL-C level. ${ }^{26,28}$ The usefulness of non-HDL Cholesterol for IHD subject and its role in the management of Diabetic dyslipidemia has been published. ${ }^{28}$

There are various studies comparing concentrations of LDL-C obtained from direct as well as calculated methods. There exists contradiction. While on one hand some studies have reported that calculated LDL-C were lower when compared to direct LDL-C, others went on to observe exactly the opposite. ${ }^{29-33}$ Similarly Direct LDL homogeneous assays are not free from limitations either. They exhibit a negative bias as observed in studies. ${ }^{14,15}$ A study has also concluded that error in Calculated LDL increases with increase in TGs. ${ }^{34}$

\section{Conclusion}

The Friedewald's estimation for LDL-C can be used for screening of patients as high or low cardiac risk groups. The direct LDL-C assay is an expensive method besides it has been observed to classify more patients in the low risk group. This can contribute in poor management, increased morbidity and mortality. Therefore the current study concludes that FF could be used to estimate LDL-C and direct LDL estimation should be employed only in those cases where in FF cannot be used like non fasting samples, patients with TGs more than $400 \mathrm{mg} / \mathrm{dL}$, disorders related to lipoproteins. Larger data is required to explain the low values obtained by direct LDL-C estimation method are comparable to Friedewald's estimation and non-HDL Cholesterol. 
Acknowledgements: The authors are grateful to Dept. of Medicine and technical staff of the Clinical Biochemistry Laboratory.

\section{Conflict of Interest: Nil}

\section{References}

1. Anderson KM, Castelli WP, Levy D. Cholesterol and Mortality: 30 years of follow-up from the Framingham Study. The journal of American medical association. 1987;257:2176-2180.

2. Gordon T, Kennel WD, Castelli WP, Dauber TR. Lipoproteins, Cardiovascular disease and death. The Framingham Study. Archives of internal medicine. 1981;141:1128-1131.

3. Third Report of the National Cholesterol Education Program (NCEP) Expert Panel on Detection, Evaluation, and Treatment of High Blood Cholesterol in Adults (Adult Treatment Panel III) final report. Circulation. 2002;106:3143-3421.

4. Ana V, Jelena KS, SlavicaS, Nada B, Jelena M, Milica V, Vesna SK, Aleksandra Z, Dragoljub P. Evaluation of different formulas for LDL-C calculation. Lipids in health and disease. 2010;9:27.

5. Third Report of the National Cholesterol Education Program Expert Panel on Detection, Evaluation and Treatment of High Blood Cholesterol in Adults (Adult Treatment Panel III). Executive summary. The journal of American medical association. 2001;285:2486-2497.

6. Fei H, Maeda S, Kirii H, Fujigaki S. Evaluation of Two Different Homogenous Assays for LDL-Cholesterol in Lipoprotein-X Positive Serum. Clinical Chemistry. 2000;46:1351-1356.

7. Rifai N. Lipoproteins and Apolipoproteins. Composition, metabolism and association with coronary heart disease. Archives of Pathology and Laboratory Medicine. 1986;110:694-701.

8. Turkalp I, Cil Z, OzkazancD. Analytical performance of a direct assay for LDL-cholesterol: A comparative assessment versus Friedewald's formula - original investigation. Anadolu Kardiyol Derg. 2005;5:13-17.

9. Friedewald WT, Levy RI, Fredrickson DS. Estimation of the concentration of low-density lipoprotein cholesterol in plasma without use of the preparative ultracentrifuge. Clinical Chemistry. 1972;18:499-502.

10. Allain CC, Poon LS, Chan CSG, Richmond W, Fu PC. Enzymatic determination of total serum cholesterol. Clinical Chemistry. 1974;20:470-475.

11. Mc. Gowan MW, Artiss JD, Standberg DR, Zak B. A peroxidase coupled method for colorimetric determination of serum triglycerides. Clinical Chemistry. 1983;29(3):538-542.

12. Naito HK, Park IK. Performance characteristics of the new generation, direct, automated high-density lipoprotein cholesterol method. Clinical Chemistry. 1997;43:256.

13. Rifai N, Iannotti E, DeAngelis K, Law, T. Analytical and Clinical performance of homogenous enzymatic LDL Cholesterol assay compared with ultracentrifugation dextran sulphate $\mathrm{Mg}^{++}$method. Clinical Chemistry. 1998;44:1242-1250.

14. Yu HH, Markowitz R, De Ferranti SD, Neufeld EJ, Farrow G, Bernstein HH, Rifai N. Direct measurement of LDL cholesterol in children: Performances of two surfactant-based methods in general pediatric population. Clinical Biochemistry. 2000;33(2):89-95.
15. Harris N, Neufeld EJ, Neuberger JW. Analytical performance and Clinical utility of a direct LDLCholesterol assay in a hyperlipidemic pediatric population. Clinical Chemistry. 1996;42:1182-1188.

16. Nauck M, Rifai N. Analytical Performance and Clinical efficacy of three routine procedures for LDL Cholesterol measurement compared with the ultracentrifugationdextransulfate- $\mathrm{Mg}^{++}$method. Clinica Chimica Acta. 2000;294(1):77-92.

17. Bethesda MD, Nauck M. National Cholesterol Education Program. Recommendations on Lipoprotein measurement. Working Group on Lipoprotein measurement. Methods for LDL-C with emphasis on Homogenous Assays. NIH Publication. 1995;95:3C44.

18. Sakaue T, Hirano T, Yoshino G, Sakai K, Takeuchi H, Adachi M. Reactions of direct LDL cholesterol assays with pure LDL fraction and LDL: Comparison of three Homogenous methods. Clinica Chimica Acta. 2000;295(1):97-106.

19. Castelli WP, Garrison RJ, Wilson PW, Abbott RD, Kalousdian S, Kammel WB. Incidence of Coronary Heart Disease and Lipoprotein levels. The Framingham Study. The journal of American medical association. 1986;256:2835-2838.

20. Randomized Trials of Cholesterol lowering in 4444 patients with Coronary Heart Disease: The Scandinavian Simvastin Survival Study (4S). Lancet. 1994;344:13831389.

21. Warnick GR, Knopp RH, Fitzpatrick V, Branson L. Estimating low density lipoprotein cholesterol by Friedewald equation is adequate for classifying patients on the basis of nationally recommended cut points. Clinical Chemistry. 1990;36:15-19.

22. Marniemi J, Maki J, Maatela J, Jarvisalo J, Impivaara O. Poor applicability of Friedewald formula in the assessment of serum LDL- Cholesterol for Clinical purposes. Clinical Biochemistry. 1995;28:285-289.

23. Nauck M, Wanick GR, Rifai N. Methods of Measurement of LDL- Cholesterol: A Critical Assessment of Direct Measurement by Homogenous Assays versus Calculation. Clinical Chemistry. 2002;48(2):236-254.

24. Miller WG, Waymack PP, Anderson FP, Ethridge SF, Jayne EC. Performance of four homogenous Direct Methods for LDL- Cholesterol. Clinical Chemistry. 2002;48(3):489-498.

25. Hsia SH. Editorial: Non- HDL Cholesterol: Into the Spotlight. Diabetes Care. Alexandria. 2003;26(1):240242.

26. Whiting MJ, Shepard MD, Tallis GA. Measurement of plasma LDL- Cholesterol in patients with diabetes. Diabetes Care. 1997;20:12-14.

27. Lu W, Resnick HE, Jablonski KA, Jones KL, Jain AK, Howard WJ, Robbins DC, Howard BV. Non- HDL Cholesterol as a predictor of Cardiovascular Disease in Type 2 Diabetes: the Strong Heart Study. Diabetes Care. 2003;26:16-23.

28. Sudha K, Prabhu KA, Hegde A, Marathe A, Kumar KA. Effect of serum triglycerides on LDL estimation by Friedewald formula and direct assay: A laboratory based study. International Journal of Biomedical Research. 2015;6:189-191.

29. Boshtam M, Ramezani MA, Naderi G, Sarrafzadegan N. Is Friedewald formula a good estimation for low density lipoprotein level in Iranian population? Journal of Research in Medical Sciences. 2012;17:519-522. 
30. Kapoor R, Chakraborty M, Singh N. A leap above Friedewald formula for calculation of low density lipoprotein cholesterol. Journal of Laboratory Physicians. 2015;7:11-16.

31. Martin SS, Blaha MJ, Elshazly MB, Brinton EA, Toth PP, McEvoy JW. Friedewald estimated versus directly measured low density lipoprotein cholesterol and treatment implications. Journal of American College of Cardiology. 2013;62:732-739.

32. Kannan S, Mahadevan S, Ramji B, Jayapaul M, Kumaravel V. LDL-cholesterol: Friedewald calculated versus direct measurement study from a large Indian laboratory database. Indian Journal of endocrinology and metabolism. 2014;18:502-504.

33. Nakanishi N, Matsuo Y, Yoneda H, Nakamura K, Suzuki $\mathrm{K}$, Tatara K. Validity of the conventional indirect methods including Friedewald method for determining serum low density lipoprotein cholesterol level: Comparison with the direct homogeneous enzymatic analysis. Journal of Occupational Health. 2000;42:130137.
34. Teerakanchana T, Puavilai W, Suriyaprom K,

Tungtrongchitr R. Comparative study of LDL cholesterol levels in Thai patients by the direct method and using the Friedewald formula. The Southeast Asian Journal of Tropical Medicine and Public Health. 2007;38:519-527.

How to cite this article: Chatterjee B.P, Sendhav S, Kakaiya A, Biswas P. C. A comparative analysis of direct LDL-C assay and Friedewald's formula in subjects of ischemic heart disease \& stroke in a tertiary care centre. Int $\mathrm{J}$ Clin Biochem Res. 2018;5(4):541-546. 